\title{
Thyroid Hormones and Mitochondrial Development in Skeletal Muscle of Fetal Sheep near term
}

\section{K.L. Davies ${ }^{1}$, A.J. Forhead ${ }^{1}$, M.J. De Blasio ${ }^{1}$, A.J. Murray ${ }^{1}$ and A.L. Fowden ${ }^{1}$ \\ Department of Physiology, Development and Neuroscience, University of Cambridge, Cambridge, CB2 3EG, UK}

\section{Introduction}

The fetal to neonatal transition is associated with an increase in oxygen consumption ${ }^{1}$. Skeletal muscle accounts for $30 \%$ of fetal oxygen uptake ${ }^{2}$, and requires increased energy at birth as it takes on the roles of thermogenesis and locomotion.

Aerobic ATP production is regulated by mitochondria (Fig.1).

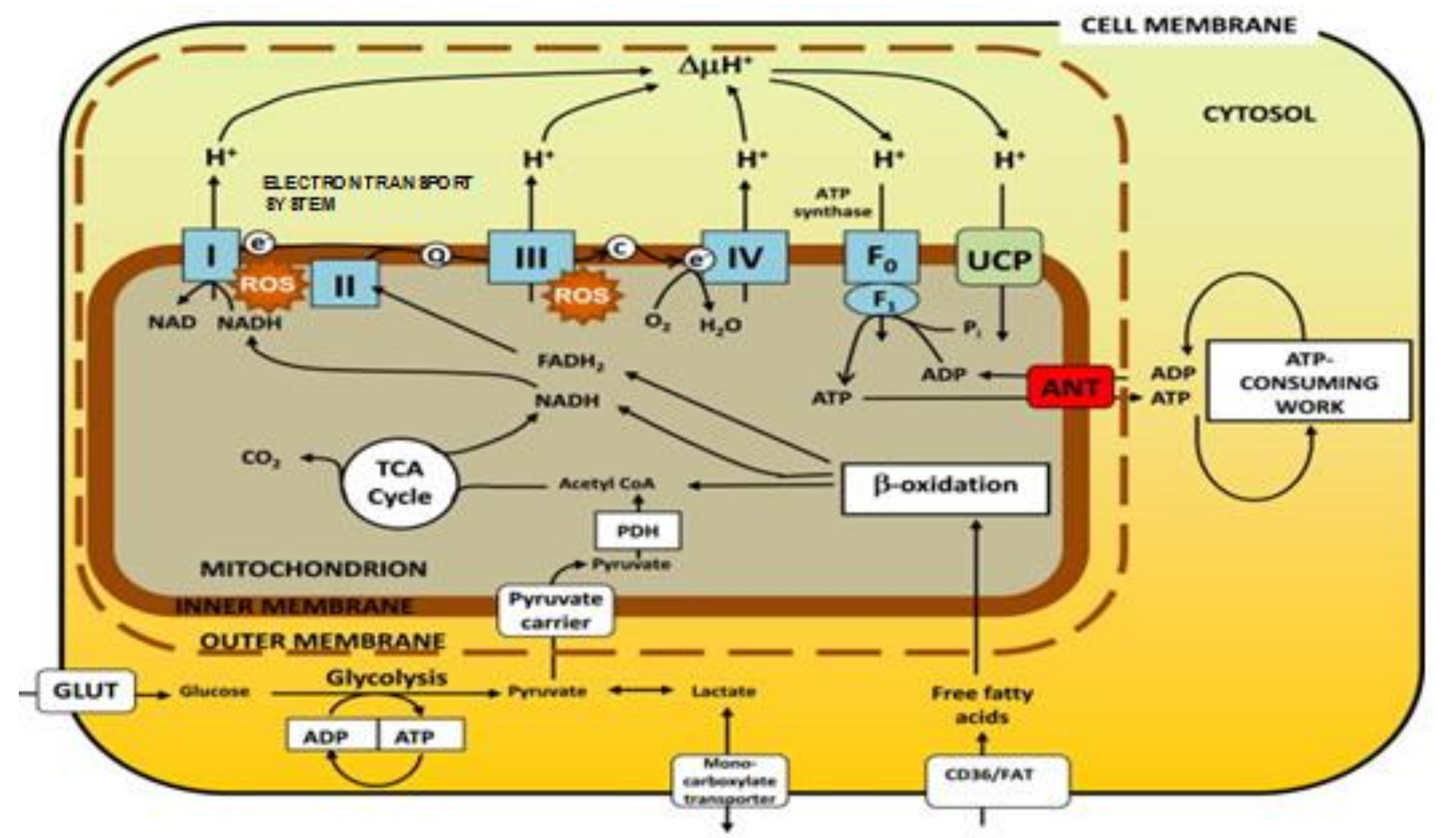

Figure 1. Diagram showing the mitochondrial pathways

involved in oxidative metabolism

In adult tissues, thyroid hormones increase mitochondrial biogenesis and oxidative metabolism through their influence on enzyme abundance, activity and proton leak ${ }^{5}$. In the fetus, thyroid hormones influence total oxygen consumption ${ }^{2}$, as well as maturation of several fetal tissues including skeletal muscle when fetal triiodothyronine $\left(T_{3}\right)$ concentration rises towards term ${ }^{6}$. However, whether thyroid hormones regulate any changes in fetal mitochondrial function during late gestation is unknown.

\section{Methods}

- After 11 twin-bearing Welsh Mountain ewes were anaesthetised at 105 days of gestation ( $\mathrm{d}$; term $\sim 145 \mathrm{~d}$ ) one fetus was thyroidectomised (TX) and its twin was sham-operated. All procedures were carried out under the Animals (Scientific Procedures) Act 1986 after ethical approval of the University of Cambridge.

- At $129 d$ ( $n=6$ ewes) and $143 d$ ( $n=5$ ewes), the ewes were killed with an overdose of anaesthetic $(200 \mathrm{mg} / \mathrm{kg}$ sodium pentobarbitone i.v.). Fetal plasma samples were taken before both fetuses were killed $(200 \mathrm{mg} / \mathrm{kg}$ sodium pentobarbitone i.v.) and biceps femoris muscle samples were dissected, weighed and immediately frozen in liquid nitrogen. Fetal plasma triiodothyronine $\left(T_{3}\right)$ concentrations were measured using radioimmunoassays.

- Muscle citrate synthase activity was measured spectrophotometrically as an index of mitochondrial density ${ }^{7}$

- Abundance of electron transport system (ETS) complexes I-IV and ATPsynthase was measured by western blotting

- Results are presented as mean \pm SEM. Data were analysed using Pearson correlation coefficient, and compared using a 2-way ANOVA followed by Tukey's post-hoc test. $P<0.05$ was considered significant.

\section{Results}

Skeletal muscle citrate synthase activity was higher at $143 d$ than 129d (Fig.2). At both ages, muscle from TX fetuses had a lower citrate synthase activity than the control muscle (Fig.2).

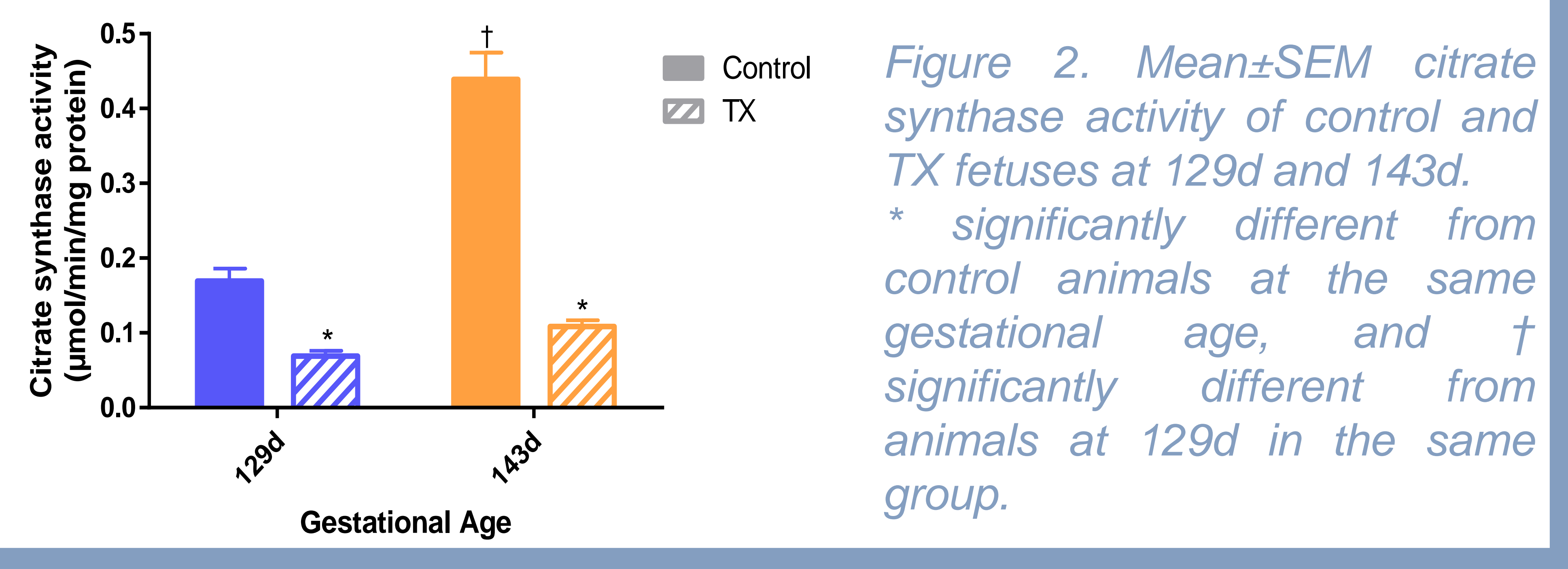

Combining all data, muscle citrate synthase activity showed a positive correlation with fetal plasma $\mathrm{T}_{3}$ concentration (Fig.3).

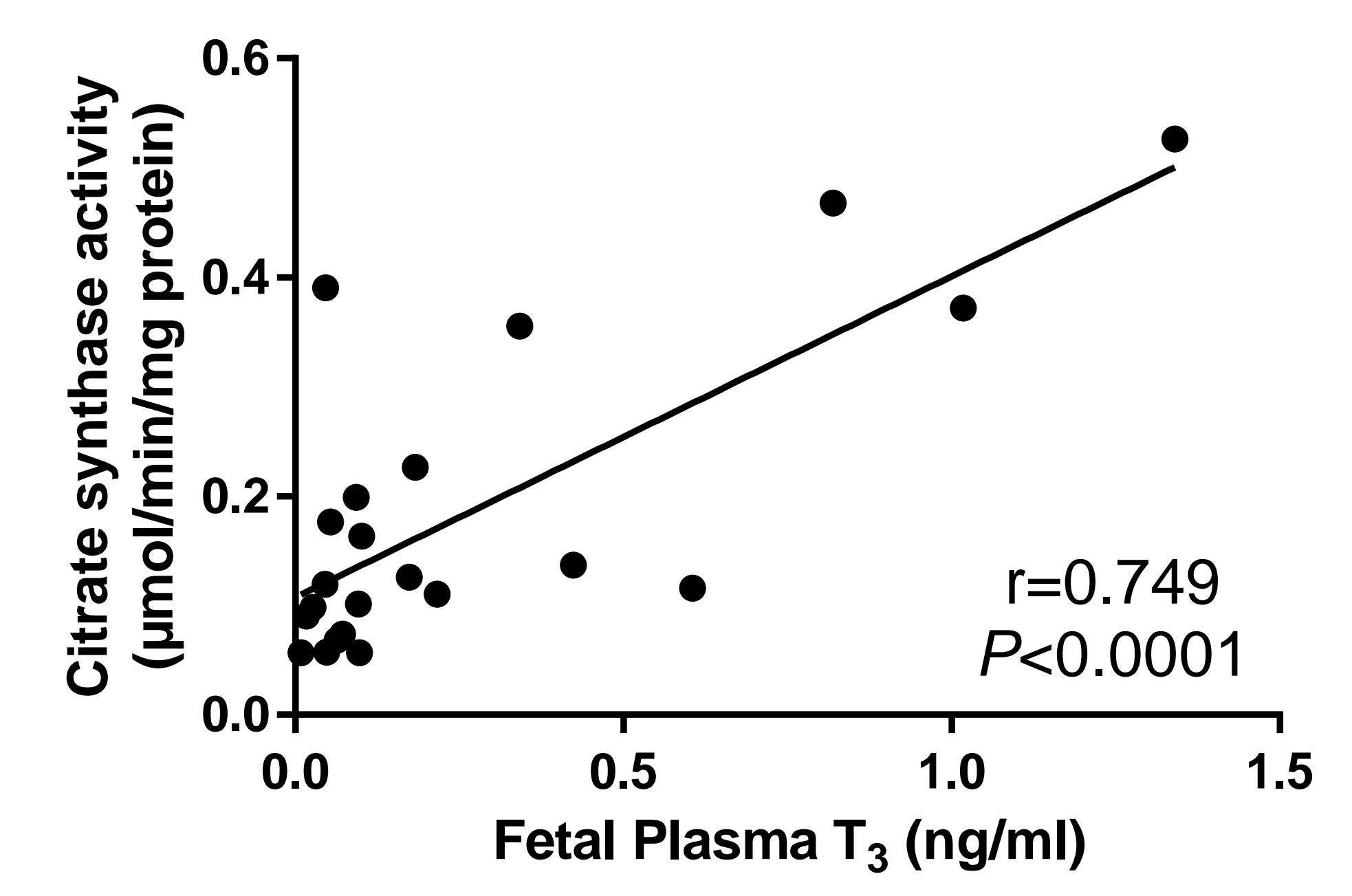

Figure 3. Correlation of citrate synthase activity in muscle with fetal plasma $T_{3}$ concentration.

Protein abundance of ETS complexes I-IV and ATP-synthase were lower in muscle from TX fetuses than the age-matched controls, with the difference being significant for complexes I and IV at 129d and for ATP-synthase and ETS complexes I, III and IV of 143d (Fig.4).
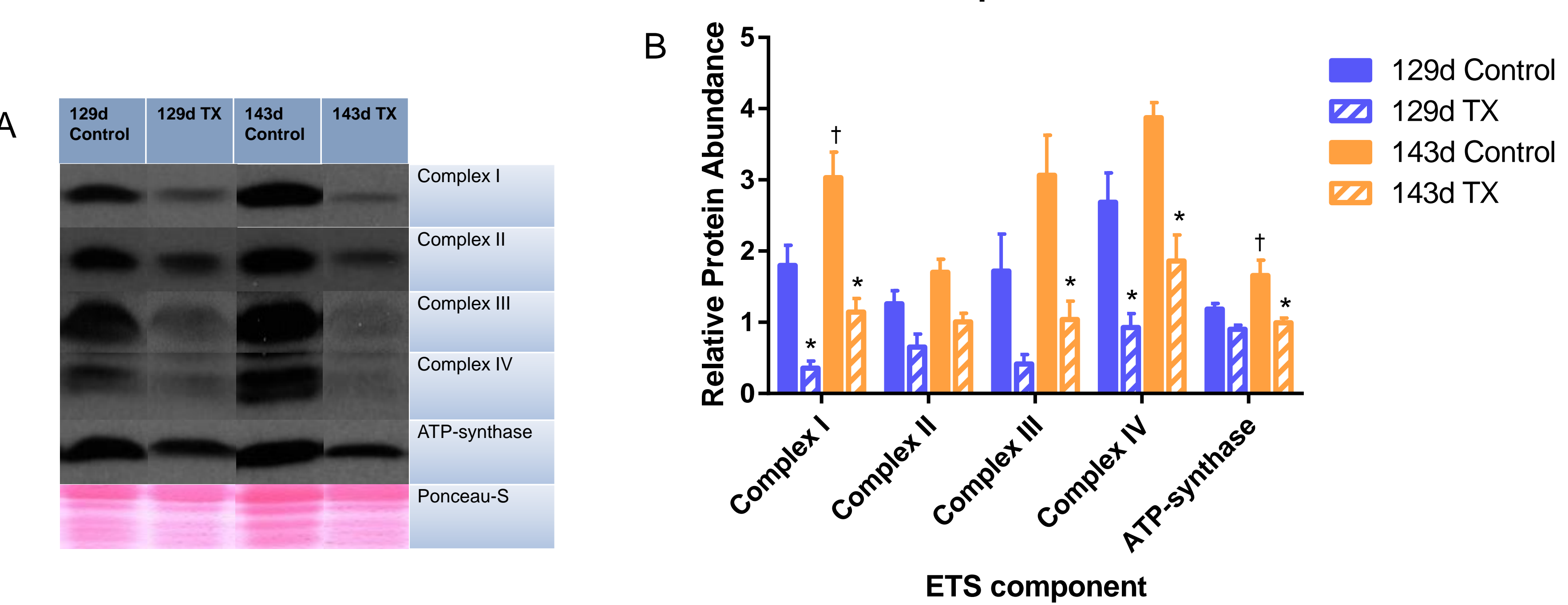

Figure 4. Protein abundance of ETS complexes I-IV and ATP-synthase.

A) representative western blot and Ponceau-S staining loading control. B Mean \pm SEM protein abundance of control and TX fetuses at 129d and 143d. significantly different from control animals at the same gestational age, and significantly different from animals at 129d in the same group.

As an indication of protein abundance per mitochondrion, protein levels of the ETS complexes and ATP-synthase were normalised to CS activity. ATP-synthase per mitochondrion was consistently higher in muscle from TX fetuses than from the age-matched controls (Fig.5).
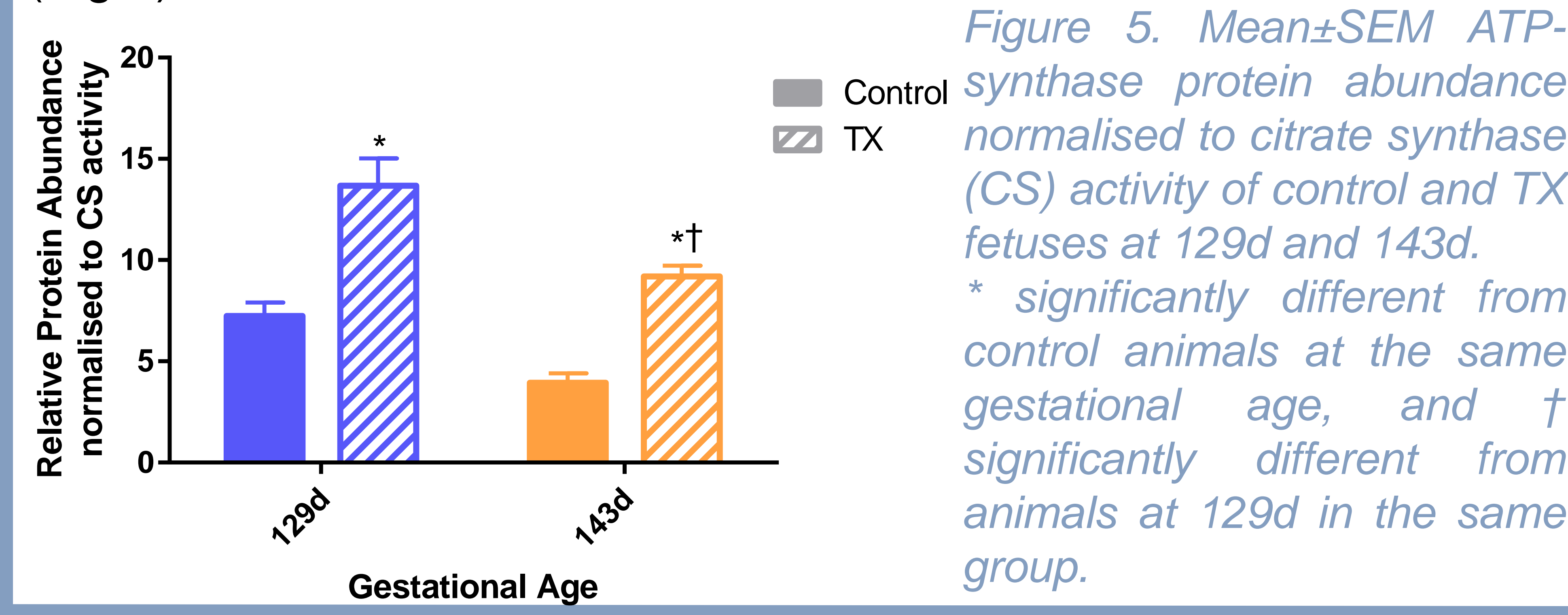

\section{Conclusions}

- Thyroid hormones are necessary for the normal developmental increase in mitochondrial density and components of oxidative phosphorylation in ovine biceps femoris in the lead-up to birth. Future work will determine whether the tissue respiratory function in situ correlates with these in vitro findings.

- ATP-synthase levels per mitochondrion are higher in muscle from hypothyroid fetuses, suggesting a compensatory response to maintain ATP production with fewer mitochondria.

\section{Acknowledgements and References}

This work was supported by the Wellcome Trust.

1. Klein et al. (1983) Am J Physiol 244:E603 2. Fowden and Silver (1995) J Physiol (Lond) 482:203 3. Rogers et al. (1998) Br J Obstet Gynaecol 94:120 4. Murray (2012) Placenta 33 (Suppl 2):e16 5. Cioffi et al. (2013) Mol Cell Endocrinol 379:51 6. Forhead and Fowden (2014) J Endocrinol 221:R87 7. Larsen et al. (2012) J Physiol 590:3349 